\title{
What is the real nature of blinkers?
}

\author{
D. Marik and R. Erdélyi
}

\author{
Space \& Atmosphere Research Center, Dept. of Applied Mathematics, University of Sheffield, Hicks Building, \\ Hounsfield Road, Sheffield, S3 7RH, England, UK \\ e-mail: Robertus@sheffield.ac.uk
}

Received 14 May 2002 / Accepted 23 August 2002

\begin{abstract}
In the present paper a simple physical model of blinkers based on the process of magnetic reconnection is developed. Blinkers were first found in transition region lines (e.g. He I, O III, O IV, O V and Mg IX) by SOHO CDS. Their typical lifetime is approximately $16 \mathrm{~min}$, the intensity enhancement ratios are around 1.8, and they appear at $1-20 \mathrm{~s}^{-1}$ on the solar disk. According to observations, blinker events seem to be increases in density and/or filling factor rather than increases in temperature. Most blinkers have a repetitive nature and a high percentage of these events occur above unipolar magnetic fields. Magnetic reconnection in the lower transition region of the solar atmosphere is numerically simulated by solving the fully nonlinear, time-dependent, dissipative, radiative 2D MHD equations. Setting the initial parameters describing transition region explosive events as in Roussev et al. (2001a,b,c), we computed the evolution of the reconnection jets. Taking into account the limit of the spatial and temporal resolution of the CDS camera and converting the high-resolution numerical results into "CDS-resolution", the propagating reconnection jets are found to have similar properties as those described by CDS blinker observations. These results suggest SOHO CDS may actually observe reconnection driven explosive events as blinkers.
\end{abstract}

Key words. Sun: atmosphere - transition region - evolution - magnetic fields - magnetohydrodynamics - numerical

\section{Blinkers observations}

Transition region blinkers (TRBs) are controversial phenomena observed in the past five years in the lower solar atmosphere. Numerous blinker observations followed their discovery by the Coronal Diagnostic Spectrometer (CDS) of the Solar and Heliospheric Observatory (SOHO) (Harrison 1997).

Blinkers are best detected in the $\mathrm{O} v$ line and are found to occur in both active and quiet regions of the Sun. Typical behaviour of active-region blinkers is very similar to quiet-region blinkers (for more details see e.g. Bewsher et al. 2002).

The mean lifetime of a blinker event is approximately $16 \mathrm{~min}$, the mean rise and fall times are around $8 \mathrm{~min}$ and the intensity enhancement ratios are around 1.8 . There is evidence that blinkers appear to be increased in density and/or filling factor rather than increased in temperature. Harrison et al. (1999) suggest it is probable that blinker events are driven by activity which most likely continues to dump energy into the plasma throughout the blinker duration. Since temperature changes may take place on time scales comparable or smaller than the ionization and recombination time scales of the plasma, timedependent ion populations need to be considered (Sarro et al. 1999; Teriaca et al. 1999).

The mean area of blinkers is $2.9 \times 10^{7} \mathrm{~km}^{2}$. The frequency of blinker events on the solar disk is $1-20 \mathrm{~s}^{-1}$. Most blinkers

Send offprint requests to: D. Marik,

e-mail: D.Marik@sheffield.ac.uk have repetitive nature though according to their Fourier and wavelet analysis no evidence of any periodicity has been found so far. The statistical analysis of blinkers also shows their distribution is not uniform: they are located in regions of strongest emission in the chromospheric and TR lines. In addition, almost all blinkers occur above well defined strong magnetic fragments and approx. $80-90 \%$ of this events can be found above regions where only one polarity dominates.

It is intriguing to note the lack of simultaneous correlation between brightenings in high-temperature lines (e.g. Mg IX) compared to cooler lines which could indicate the corona is at least partially decoupled from the TR and cromosphere (Brković et al. 2001).

\section{Blinkers as CDS signature of reconnection?}

We propose a blinker model, coinciding best with the above observational findings, based on magnetic reconnection. Our aim is to investigate the plasma jets driven by the process of magnetic reconnection in a $2 \mathrm{D}$ physical environment representing the solar TR. Magnetic flux cancellation may be initiated by localized increase of magnetic diffusivity in a current concentration separating two magnetic flux tubes (Roussev et al. 2002). Another driving mechanism could be the reconnection between randomly emerging magnetic flux at the boundary of active regions or supergranular cells where the newly emerged flux is pushed into the background magnetic field (Jin et al. 1996). In both cases, reconnection results in jet ejections along the 
current sheet developed at magnetic shears. (Note it is not necessary to have opposite polarities! See, e.g. Zhang et al. 2000; Ryutova \& Tarbell 2000.)

The above picture of reconnection is accepted as a probable mechanism driving explosive events (Innes et al. 1997; Roussev et al. 2001b). Explosive events (EEs) are strongly localised high velocity jets in the transition region observed by SUMER onboard SOHO. How could they link to slow speed TRBs observed by CDS? What could their role be in blinker evolution? In spite of the apparently distinctive character of these two phenomena we propose they may be manifestations of the same physical process observed by different instruments. CDS has, at most, a spatial resolution of $1.7^{\prime \prime} \times 4.0^{\prime \prime}$ which is many times the area of an average explosive event on the solar surface. Hence, CDS provides unresolved integrated signals from a whole surface where many tiny and localised magnetic reconnections may occur. This may suggest blinkers could in reality be identical to EEs (or a series of them) but CDS cannot resolve these events. In what follows we elaborate on this and give a typical numerical example. MHD reconnection simulations of EEs are carried out. The reconnection jet velocity and profiles are re-mapped into CDS resolutions. These re-mapped profiles are found not be in contradiction with observations describing TRBs.

\section{Numerical results}

\subsection{The equilibrium model}

The solar atmospheric plasma is considered as an ideal gas embedded in an intermittent inhomogeneous vertical magnetic field ( $y$-coordinate), where $x z$ is the plane of the solar surface with $z$-coordinate being the ignorable direction (see Fig. 1). Gravity is neglected. The governing equations of a 2D dissipative, radiative MHD are considered (see, e.g. Roussev et al. $2001 \mathrm{~b}$ for details on inclusion of dissipation and radiation).

The initial magnetic field configuration describing a current sheet is given by

$\boldsymbol{B}=\left(0, B_{y}(x), 0\right) ; \quad B_{y}=B_{0} \tanh \left(C x / L_{0}\right)$,

where $L_{0}$ and $B_{0}$ are typical values of length scale and magnetic field strength, respectively, and $C$ is a constant. The initial equilibrium velocity is assumed to be zero throughout the computational domain. The total pressure balance is given by

$$
\frac{B_{y}^{2}(x)}{2}+P(x)=\frac{B_{0}^{2}}{2}+P_{0}=\text { const. }
$$

where $P(x)$ is the kinetic gas pressure and can be computed once the initial magnetic field distribution, $B_{y}(x)$ is given. For the sake of simplicity a uniform kinetic gas pressure distribution in the $y$-direction is assumed as a first approximation resulting in the thermal energy, $e=P /(\gamma-1)$, also being uniform along $y$. From the total pressure balance it follows that

$$
e(x, y)=e(x)=\frac{e_{0}}{\beta}\left[1+\beta-\left(\frac{B_{y}(x)}{B_{0}}\right)^{2}\right] .
$$
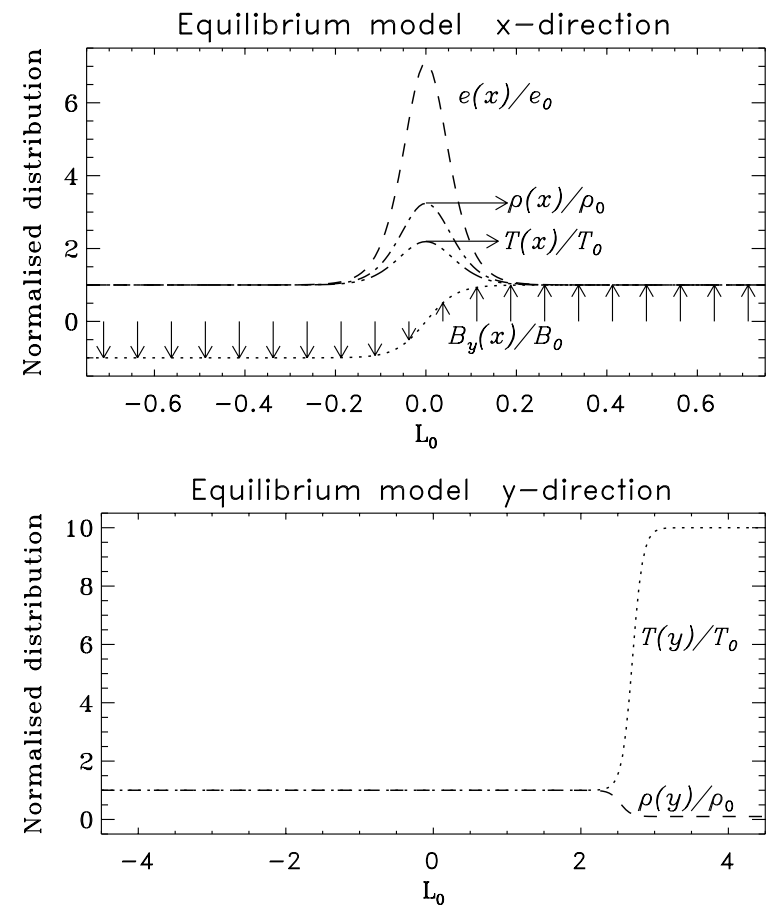

Fig. 1. The equilibrium stratification of the initial magnetic field, pressure, temperature and density.

To represent the TR containing a current sheet the density stratification in the $y$-direction is given by

$\varrho(y)=\varrho_{0}\left\{1+\Delta \varrho\left\{1-\tanh \left[\frac{\varepsilon}{L_{0}}\left(y+y_{\mathrm{s}}\right)\right]\right\}\right\}$,

where $\varrho_{0}$ is a typical value of the mass density in the low density region and $2 \Delta \varrho$ is the jump in density across the transition region. $\varepsilon$ is a steepness parameter and $y_{\mathrm{s}}$ is a shifting parameter describing the center of the density jump relative to the photosphere. The corresponding equilibrium temperature profile is an increasing function in height, and is given by $T(y)=P / \varrho(y)$. The mass density distribution in the $x$-direction reads as

$\varrho(x)=\varrho_{0}\left(\frac{P}{P_{0}}\right)^{\theta}=\varrho\left(\frac{e}{e_{0}}\right)^{\theta}$,

where $\theta$ is a free parameter (one over the polytropic index). The full $2 \mathrm{D}$ profile of the mass density is computed by $\varrho(x, y)=$ $\varrho(x) \varrho(y) / \varrho_{0}$.

Using the equation of state $e=\varrho T /(\gamma-1)$, one can derive the 2D temperature profile $T(x, y)$. Finally we also assume energy balance in the entire physical domain for the initial state: $S(x, y)-\nabla \boldsymbol{q}(x, y)-L_{\mathrm{r}}(x, y)=0$ at $t=0$, where $S, \boldsymbol{q}$ and $L_{\mathrm{r}}$ are the volumetric heating rate, the heat flux and the radiative loss, respectively.

Magnetic reconnection is governed by a time-dependent localized magnetic diffusion

$\eta_{\text {loc }}=\eta_{0} F(t) \exp \left\{-\frac{\alpha}{L_{0}^{2}}\left[x^{2}+\left(y+y_{0}\right)^{2}\right]\right\}$,

where $F(t)$ is a function of time, $\alpha$ is a steepness parameter, $y_{0}$ is a shifting coefficient and $\eta_{0}=V_{\mathrm{A} 0} L_{0} / R_{\mathrm{m}}\left(R_{\mathrm{m}}\right.$ is the magnetic Reynolds number, $V_{\mathrm{A} 0}$ is the Alfvén speed given by $\left.V_{\mathrm{A} 0}=B_{0} / \sqrt{\varrho_{0}}\right)$. 

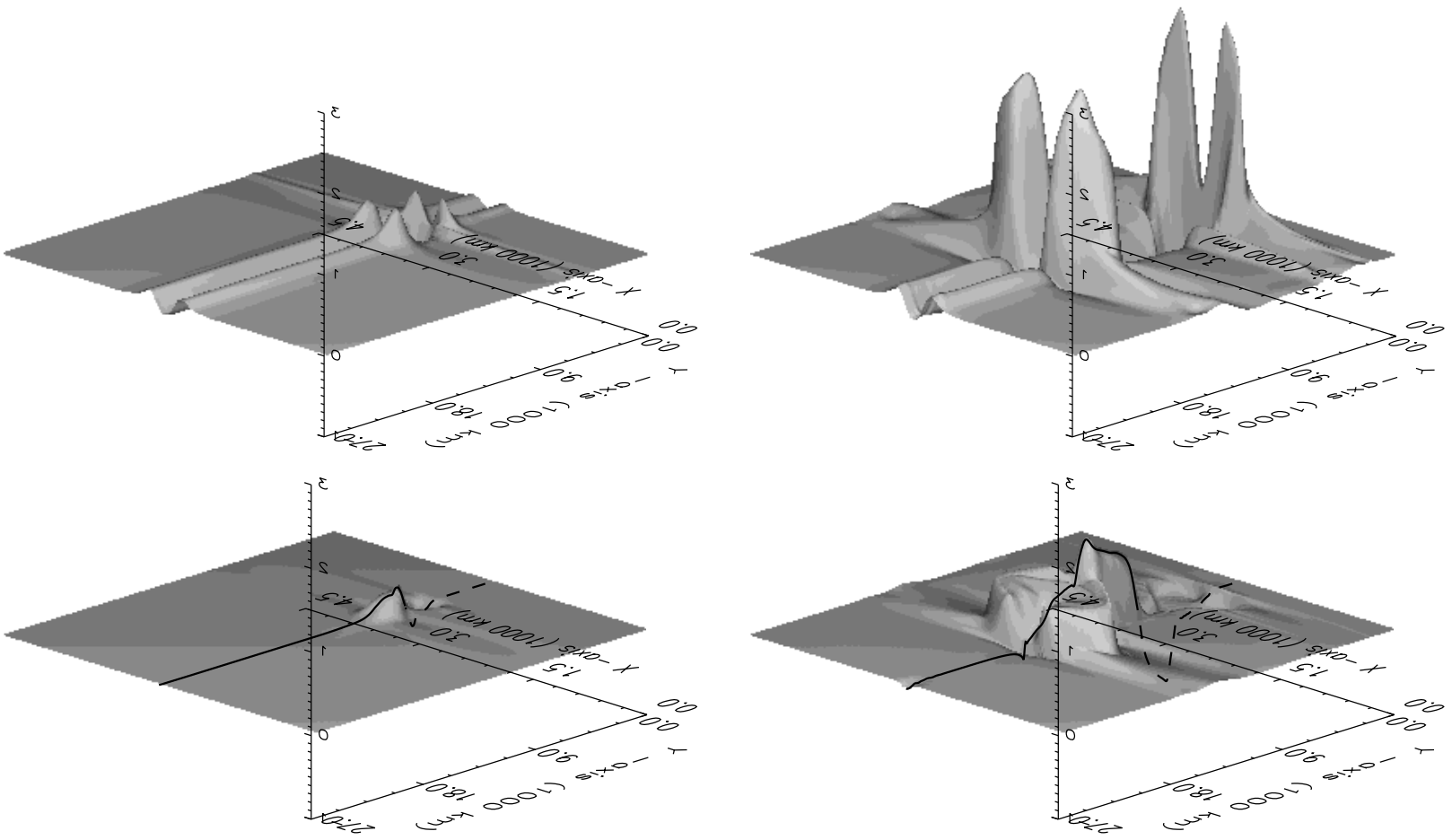

Fig. 2. Time evolution of the relative density (top) and velocity (bottom) distributions of the reconnection jets. The density is in units of $\varrho_{0}=2.54 \times 10^{-14} \mathrm{~g} \mathrm{~cm}^{13}$. The velocity is in units of Alfvén speed $\left(V_{\mathrm{A} 0}=1.293 \times 10^{2} \mathrm{~km} \mathrm{~s}^{-1}\right)$. The real times of the images are $205 \mathrm{~s}($ left $)$, and $334 \mathrm{~s}$ (right). Domain size: $y \in\left[-4.5 L_{0}, 4.5 L_{0}\right]$ and $x \in\left[-0.75 L_{0}, 0.75 L_{0}\right]$ where $L_{0}=3000 \mathrm{~km}$.

\subsection{Reconnection jets}

The compressible fully nonlinear 2D MHD equations are solved using non-uniform staggered grids (Nordlund et al. 1995) for typical parameters of EEs. Our aim here is not to study the parameter space for EEs; this has recently been extensively done by Roussev et al. (2001a,b,c, 2002), instead we focus on obtaining characteristic profiles of velocity, density and temperature distributions for EEs. These profiles will be re-mapped into CDS resolution and will be compared to TRBs.

When the localized magnetic diffusion parameter is switched on, representing either emerging flux or anomalous resistivity, magnetic field on the sides of the formed current concentration begins to reconnect. This process changes the local topology of the magnetic field and magnetic flux starts to advect into a so-called $\mathrm{X}$-point along the $x$-direction, and is expelled from the diffusion region through two reconnection jets along the $y$-axis. Figure 2 shows there is a blue shifted jet propagating towards the high temperature region $\left(y>y_{0}\right)$ with increasing velocity and there is a red shifted jet moving towards the high density region $\left(y<y_{0}\right)$ submerging into the lower atmosphere. The jet heads reach the TR Alfén velocity within a few minutes (Fig. 2 bottom row). They also show local density enhancements higher than values derived from blinker observations (top row). For a typical EE the enhanced magnetic diffusion is switched off after around $40 \mathrm{~s}$ (average life time of explosive events).

EEs simulated above (or seen by SUMER) appear to have different properties from blinker events seen by CDS as described above. They are smaller and their lifetime is much
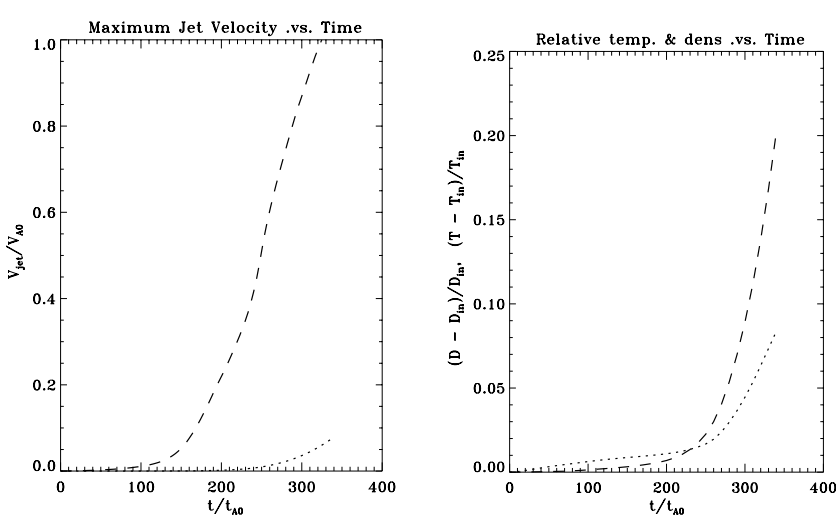

Fig. 3. Left are the simulated (long dashed) and the re-mapped "CDSeye" integrated (dotted) velocity profiles obtained from the case study in Fig. 2. $\left(V_{\mathrm{A} 0}=1.293 \times 10^{2} \mathrm{~km} \mathrm{~s}^{-1}, t_{\mathrm{A} 0}=23.2 \mathrm{~s}\right)$. Right are the corresponding relative density (long dashed) and the relative temperature (dotted) profiles.

shorter. The observed velocity profiles also appear not to match, because EEs have a maximum jet head velocity around the TR Alfvén speed (as it should be!) whilst, according to recent spectral analyses TBRs do not really show considerable velocity enhancements. Blinker velocities of around $20 \mathrm{~km} \mathrm{~s}^{-1}$ are the upper limit (Harrison et al. 1999).

\subsection{Looking at explosive events by "CDS-eyes"}

Let us resolve these apparent contradictions. When carrying out numerical simulations we can sample and visualize our 
data at high resolution. (In theory one is limited only by processor and round-off errors.) Let us now re-map the obtained high spatial and temporal resolution results of the reconnection model of EEs in Fig. 2 by taking into account the resolution limits of a typical CDS observation of TRBs. We convert the velocity, temperature and density profiles of the outwards propagating blue jet of an EE. Before we proceed we recall: (i) a CDS pixel overlaps a $1.7^{\prime \prime} \times 4^{\prime \prime}$ domain which actually corresponds to more than the whole EE area on the solar surface; (ii) the CDS exposure time is around $10 \mathrm{~s}$; (iii) images are averaged over the whole depth of a domain. Taking into account all these features we construct mean values of the numerically determined velocity, temperature and density along the $x$-axis at every point of the $y$-axis when re-mapping. In the model, the domain along the $x$-axis was $\left[-0.21 L_{0}, 0.21 L_{0}\right]$ and was symmetric about the $y$-axis. This domain corresponds to approx. $1.7^{\prime \prime}$ on the solar surface. The next step is to take a mean value along the $y$-axis and finally we have to average snapshots in time by integration for each $10 \mathrm{~s}$ interval (cadence). This method is a simplified procedure to include the effects of how, through a CDS pixel, one would "see" if the line-of-sight coincides with the direction of the jet propagation, i.e. the $y$-axis. Figure 3 a shows the difference between the numerical simulation (maximum speed in the line of sight) and what CDS would "see" as an integrated velocity profile.

Although there does not seem to be an obvious saturation of the integrated velocity profile around the $20 \mathrm{~km} \mathrm{~s}^{-1}$ observed maximum, it reaches much lower values than the actual simulations (Fig. 3a) and remains well under this threshold. Unfortunately we cannot simulate blinkers for longer than 6-8 min as a 6 min event takes more than two days CPU to run. However, the location of the blue jet after $300 \mathrm{~s}$ is already well in the solar corona anyway. Similarly in Fig. 3b one sees that the integrated, averaged relative temperature and density. Observe the enhanced density as speculated by Harrison (1997). These integrated velocity, relative temperature and density profiles suggest the reconnection model could describe blinkers.

On the other hand, one has to recall that $80-90 \%$ of blinkers are in unipolar magnetic field dominated areas which may contradict blinkers are the results of magnetic reconnection unless there is a shear in unipolar magnetic fields. Sunspots in many cases show light bridges providing sheared fields. There is no reason to believe that smaller flux tubes cannot have such bridges as well. Two final comments: (i) The re-mapped density enhancement of an EE per CDS pixel may seem to be small. However, EE's show up very repeatedly in SUMER lines. (ii) The averaged temperature enhancement in Fig. 3b $(\approx 10 \%)$ is well within observational errors.

We suggest a statistical analysis of joint SUMER and CDS observations supported by high-resolution ground based magnetograms to construct a magnetic carpet of blinkers may shed light on the role of explosive events in blinker formation.

Acknowledgements. The authors thank the thorough reading and comments of M. Goossens. R. Erdélyi is grateful to M. Kérai for patient encouragement, to The Nuffield Foundation (NAL/99-00) and to NSF Hungary (OTKA TO32462).

\section{References}

Bewsher, D., Parnell, C. E., \& Harrison, R. A. 2002, Sol. Phys. 206 , 21

Brković, A., Solanki, S. K., \& Rüedi, I. 2001, A\&A, 373, 1056

Harrison, R. A. 1997, Sol. Phys., 175, 467

Harrison, R. A., Lang, J., Brooks, D. H., \& Innes, D. E. 1999, A\&A, 351,1115

Innes, D. E., Inhester, B., Axford, W. I., \& Wilhelm, K. 1997, Nature, 386,811

Jin, S.-P., Inhester, B., \& Innes, D. 1996, Sol. Phys., 169, 279

Nordlund, ^, \& Galsgaard, K. 1995, A 3D MHD code for Paralell Computers, www-solar.mcs.st-adn.ac.uk/ klaus/

Roussev, I., Galsgaard, K., Erdélyi, R., \& Doyle, J. G. 2001a, A\&A, 370, 298

Roussev, I., Galsgaard, K., Erdélyi, R., \& Doyle, J. G. 2001b, A\&A, 375,228

Roussev, I., Doyle, J. G., Galsgaard, K., \& Erdélyi, R. 2001c, A\&A, 380, 719

Roussev, I., Galsgaard, K., \& Judge, P. G. 2002, A\&A, 382, 639

Ryutova, M. P., \& Tarbell, T. D. 2000, ApJ, 541, 29

Sarro, L. M., Erdélyi, R., Doyle, J. G., \& Pérez, M. E. 1999, A\&A, 351,721

Teriaca, L., Doyle, J. G., Erdélyi, R., \& Sarro, L. M. 1999, A\&A, 352, 99

Zhang, J., Wang, J., Lee, C.-Y., \& Wang, H. 2000, Sol. Phys., 194, 59 